COMMUNICATIONS IN

ANALYSIS AND GEOMETRY

Volume 14, Number 2, 249-268, 2006

\title{
Branched Covers of Hyperbolic Manifolds and Harmonic Maps
}

\author{
F. T. FARrell and P. Ontaneda ${ }^{1}$
}

\section{Introduction and statements of the results.}

Let $f: M \rightarrow N$ be a homotopy equivalence between closed negatively curved manifolds. The fundamental existence results of Eells and Sampson [5] and uniqueness of Hartmann [15] and Al'ber [1] grant the existence of a unique harmonic map $h$ homotopic to $f$. Based on the enormous success of the harmonic map technique, Lawson and Yau conjectured that the harmonic map $h$ should be a diffeomorphism. This conjecture was proved to be false by Farrell and Jones [6] in every dimension in which exotic spheres exist. They constructed examples of homeomorphisms $f: M \rightarrow N$ between closed negatively curved manifolds for which $f$ is not homotopic to a diffeomorphism. These counterexamples were later obtained also in dimension six by Ontaneda [21] and later generalized by Farrell, Jones and Ontaneda to all dimensions $>5$ [8]. In fact, in [21] and [8] examples are given for which $f$ is not even homotopic to a $P L$ homeomorphism. The fact that $f$ is not homotopic to a $P L$ homeomorphism has several interesting strong consequences that imply certain limitations of well known powerful analytic ethods in geometry [9], [10], [11], [12] (see [13] for a survey).

In all the examples mentioned above, one of the manifolds is always a closed hyperbolic manifold. Hence, both manifolds $M$ and $N$ have the homotopy type of a closed hyperbolic manifold (hence the homotopy type of a closed locally symmetric space). We call these examples of hyperbolic homotopy type.

In [2], Ardanza also gave counterexamples to the Lawson-Yau conjecture. In his examples, the manifolds $M$ and $N$ are not homotopy equivalent to a closed locally symmetric space; in particular, they are not homotopy

\footnotetext{
${ }^{1}$ The first author was partially supported by a NSF grant. The second author was supported in part by a research grant from CAPES, Brazil.
} 
equivalent to a closed hyperbolic manifold. We call these examples of nonhyperbolic homotopy type. In these examples, the map $f$ is not homotopic to a diffeomorphism and exists in dimensions $4 n-1, n \geq 2$. Hence Ardanza's result is an analogue of Farrell-Jones result [6] for examples of non-hyperbolic homotopy type. His constructions use branched covers of hyperbolic manifolds. Recall that Gromov and Thurston [14] proved that large branched covers of hyperbolic manifolds do not have the homotopy type of a closed locally symmetric space.

In this paper, we show that most of the results obtained for examples of hyperbolic homotopy type in [21], [8], [9], [10], [11], [12], [13] are also true for examples of non-hyperbolic homotopy type. We now state our main results.

First, we extend Ardanza's result to every dimension $>5$ and also with the stronger property that $f$ is not even homotopic to a $P L$ homeomorphism. This is an analogue of the result in [8] for examples of non-hyperbolic homotopy type.

Theorem 1.1. Given $\epsilon>0$ and $n>5$, there are closed $n$-dimensional Riemannian manifolds $M_{1}, M_{2}$ and a homeomorphism $f: M_{1} \rightarrow M_{2}$ such that:

1. $f$ is not homotopic to a PL homeomorphism. In particular, the unique harmonic map homotopic to $f$ is not a diffeomorphism.

2. $M_{1}$ and $M_{2}$ have $\epsilon$-pinched to -1 sectional curvatures.

3. $M_{1}$ and $M_{2}$ do not have the homotopy type of a closed locally symmetric space. In particular, they are not homotopy equivalent to a closed hyperbolic manifold.

Remark 1.2. This result is a little weaker than the result in [2] (for examples of non-hyperbolic homotopy type) and than the one in [8] (for examples of hyperbolic homotopy type): in [2], $M_{1}$ is not diffeomorphic to $M_{2}$ and in [8] $M_{1}$ is not $P L$ homeomorphic to $M_{2}$. The Theorem above states the existence of a particular map $f$ that is not homotopic to a $P L$ homeomorphism, but we do not know whether $M_{1}$ and $M_{2}$ are $P L$ homeomorphic. The missing ingredient is "differentiable rigidity" which, for examples of hyperbolic homotopy type, is provided by Mostow's Rigidity Theorem: every homotopy equivalence between hyperbolic manifolds is homotopic to a diffeomorphism (in fact an isometry). We do not know whether every homotopy equivalence between branched covers of hyperbolic manifolds is homotopic to a diffeomorphism. 
As for the case of examples of hyperbolic homotopy type, the fact that the map $f$ is not homotopic to a $P L$ homeomorphism has the following interesting consequence:

Corollary 1.3. Given $\epsilon>0$ and $n>5$, there are closed $n$-dimensional Riemannian manifolds $M_{1}, M_{2}$ and a harmonic homotopy equivalence $h: M_{1} \rightarrow$ $M_{2}$ such that:

1. $h$ is not one-to-one.

2. $M_{1}$ and $M_{2}$ have $\epsilon$-pinched to -1 sectional curvatures.

3. $M_{1}$ and $M_{2}$ do not have the homotopy type of a closed locally symmetric space. In particular, they are not homotopy equivalent to a closed hyperbolic manifold.

This Corollary can be directly deduced from Theorem 1.1 and the $C^{\infty}$ Hauptvermutung of Scharlemann and Siebenmann [24] (the proof of Corollary 1.3 appears after the proof of Theorem 1.1). Also, if Poincare's conjecture for three dimensional manifolds is true, then the map $h$ above is not even a cellular map, hence it cannot be approximated by homeomorphisms. The proof of this fact is similar to the proof for examples of hyperbolic homotopy type. For more details see [9] or [13].

In the examples provided by the Corollary above, the main obstruction to $h$ being a diffeomorphism or a homeomorphism is that $h$ is not homotopic to a $P L$ homeomorphism. We may ask then what happens if this obstruction vanishes, that is, if $h$ is homotopic to a $P L$ homeomorphism or even homotopic to a diffeomorphism. This was considered in Problem 111 of the list compiled by Yau in [28]. Here is a restatement of this problem.

Problem 111 of [28] Let $f: M_{1} \rightarrow M_{2}$ be a diffeomorphism between two compact manifolds with negative curvature. If $h: M_{1} \rightarrow M_{2}$ is the unique harmonic map which is homotopic to $f$, is $h$ a homeomorphism?, or equivalently, is $h$ one-to-one?

(This problem had been reposed in [27] as Grand Challenge Problem 3.6.) The answer to the problem was proved to be yes when $\operatorname{dim} M_{1}=2$ by Schoen-Yau [25] and Sampson [23]. But it was proved by Farrell, Ontaneda and Raghunathan [10] that the answer to this question is in general negative for dimensions $>5$. The counterexamples constructed in [10] are examples of hyperbolic homotopy type. Here, we construct counterexamples of nonhyperbolic homotopy type: 
Theorem 1.4. For every integer $n>5$ and $\epsilon>0$, there is a diffeomorphism $f: M_{1} \rightarrow M_{2}$ between a pair of closed $n$-dimensional Riemannian manifolds such that:

1. The unique harmonic map homotopic to $f$ is not one-to-one.

2. $M$ and $N$ have $\epsilon$-pinched to -1 sectional curvatures.

3. $M$ and $N$ do not have the homotopy type of a closed locally symmetric space. In particular, they are not homotopy equivalent to a closed hyperbolic manifold.

Also, in [11], we constructed examples of harmonic maps $h: M \rightarrow N$ between $\epsilon$-pinched to -1 closed Riemannian manifolds such that $h$ can be approximated by diffeomorphisms, but $h$ is not a diffeomorphism (in particular $h$ is a cellular map). The examples in [11] are examples of hyperbolic homotopy type. Here we also construct counterexamples of non-hyperbolic homotopy type:

Theorem 1.5. For every integer $n>10$ and $\epsilon>0$, there is a harmonic map $h: M_{1} \rightarrow M_{2}$ between a pair of closed $n$-dimensional Riemannian manifolds such that:

1. The harmonic map $h$ is not a diffeomorphism.

2. The harmonic map $h$ can be approximated by diffeomorphisms (in the $C^{\infty}$ topology).

3. $M_{1}$ and $M_{2}$ have $\epsilon$-pinched to -1 sectional curvatures.

4. $M_{1}$ and $M_{2}$ do not have the homotopy type of a closed locally symmetric space. In particular, they are not homotopy equivalent to a closed hyperbolic manifold.

As for examples of hyperbolic homotopy type, if Poincare's Conjecture for three dimensional manifolds is true, we can say a little more in this case (see [11] or [13]). Also, it seems that it is straightforward to verify (but we do not do this here) that in Theorems 1.1, 1.4 and Corollary 1.3 above (and may be also in Theorem 1.5) we can replace the word "harmonic" by "natural". The concept of natural map was defined by Besson, Courtois and Gallot (e.g. see [3]). These maps have very powerful dynamic and geometric properties and are also used to prove rigidity results. (For more details, see [13]). 
In [12], the results of [11] were used to construct examples of $\epsilon$-pinched to -1 closed Riemannian manifolds for which the Ricci flow does not converge smoothly to a negatively curved metric. These examples are examples of hyperbolic homotopy type. Here, we show also that the constructions used to prove Theorem 1.5 (which are analogous to the constructions in [11]) can also be used to produce examples of non-hyperbolic homotopy type of $\epsilon$-pinched to -1 closed Riemannian manifolds for which the Ricci flow does not converge smoothly to a negatively curved metric.

Remark 1.6. We say that the Ricci flow for a negatively curved Riemannian metric $h$ converges smoothly to a negatively curved metric if the Ricci flow, starting at $h$, is defined for all $t$ and converges (in the $C^{\infty}$ topology) to a well defined negatively curved (Einstein) metric. The next Theorem shows the existence of pinched negatively curved metrics for which the Ricci flow does not converge smoothly to a negatively curved metric.

Theorem 1.7. For every integer $n>10$ and $\epsilon>0$, there is a closed smooth $n$-dimensional Riemannian manifold $N$ such that:

1. $N$ admits a Riemannian metric with sectional curvatures in $[-1-$ $\epsilon,-1+\epsilon]$ for which the Ricci flow does not converge smoothly to a negatively curved metric.

2. $N$ does not have the homotopy type of a closed locally symmetric space. In particular, $N$ is not homotopy equivalent to a closed hyperbolic manifold.

The proofs of all Theorems above use the following Proposition:

Proposition 1.8. For every integer $n>5$ and $r, s>0$, there are closed connected orientable Riemannian manifolds $M, N, T, P$ such that:

1. $M$ is a n-dimensional hyperbolic manifold, $N$ and $P$ are totally geodesic $(n-1)$-dimensional submanifolds of $M$ and $T$ is a totally geodesic $(n-2)$-dimensional submanifold of $M . N$ intersects $T$ and $P$ transversally.

2. The isometry class of $N$ does not depend on $r$ (only on $n$ and $s$ ).

3. $0 \neq[N \cap T] \in H_{n-3}\left(M, \mathbb{Z}_{2}\right)$, where $[N \cap T]$ is the $\mathbb{Z}_{2}$-homology class represented by the $(n-3)$-dimensional submanifold $N \cap T$. 
4. The normal geodesic tubular neighborhood of $N$ has width $>r$ and the normal geodesic tubular neighborhood of $N \cap P$ has width $>s$.

Remark 1.9. The Proposition remains valid if we replace $N$ in item 2 by $T$. (We cannot choose both $N$ and $T$ to be independent of the widths of their normal geodesic tubular neighborhoods.) Also, we can have $T \subset P$, if we choose so.

In the section 2, we prove Proposition 1.8 and show how it implies the Theorems. The proofs of the Theorems resemble the proofs of the corresponding Theorems for the case of examples of hyperbolic homotopy type presented in [21], [8], [9], [10], [11], [12], [13]. We will refer to these papers.

\section{Proofs of the results.}

First we prove Proposition 1.8 .

Proof. of the Proposition 1.8 We use all notation from [10]. Let $\mathbf{G}, \mathbf{G}_{1}, \mathbf{G}_{2}$ be as in the proof of lemma of [10], p. 233. We have that $\mathbf{G}_{i}=R_{k / \mathbb{Q}} \mathbf{G}_{i}^{\prime}$ where $\mathbf{G}_{i}^{\prime}$ denotes the $k$-algebraic group $S O\left(f_{i}\right)$ and $f_{i}$ is the restriction of the form $f$ to the subspace of $E$ generated by $\mathcal{B}_{i}$, see[10] p.245. To be specific, choose $\mathcal{B}_{1}=\mathcal{B} \backslash\left\{e_{1}\right\}$ and $\mathcal{B}_{2}=\mathcal{B} \backslash\left\{e_{2}, e_{3}\right\}$.

For ideals $\underline{a}, \underline{b}, \underline{c}$, let $\Phi(\underline{a}, \underline{b}, \underline{c})$ be the arithmetic subgroup of $\mathbf{G}$ constructed in p. 234 of [10]. We will need the following two properties of $\Phi$. These properties can easily be checked directly from the definition.

a. $\Gamma(\underline{c}) \subset \Phi(\underline{a}, \underline{b}, \underline{c}) \subset \Gamma(\underline{a})$

b. If $\underline{a}^{\prime} \subset \underline{a}, \underline{b}^{\prime} \subset \underline{b}, \underline{c}^{\prime} \subset \underline{c}$ then $\Phi\left(\underline{a}^{\prime}, \underline{b}^{\prime}, \underline{c}^{\prime}\right)$ is a subgroup of finite index of $\Phi(\underline{a}, \underline{b}, \underline{c})$.

The following is shown in [10]:

There is an ideal $\underline{a}_{0}$ of $\mathbb{Z}$ such that for every ideal $\underline{a} \subset \underline{a}_{0}$, there is an ideal $\underline{b}(\underline{a}) \subset \underline{a}$ with the following properties. For any ideal $\underline{c} \subset \underline{b}(\underline{a})$, define $\Phi=\Phi(\underline{a}, \underline{b}(\underline{a}), \underline{c})$, and $\Phi_{i}, M, N, T$, as in p. 234 of [10]. Then $M, N$ and $T$ are closed connected orientable manifolds that satisfy:

1. $M$ is a $n$-dimensional hyperbolic manifold, $N$ is a totally geodesic $(n-1)$-dimensional submanifold of $M$ and $T$ is a totally geodesic (n-2)-dimensional submanifold of $M . N$ and $T$ intersect transversally.

2. The isometry class of $N$ does not depend on $\underline{c}$. 
3. $0 \neq[N \cap T] \in H_{n-3}\left(M, \mathbb{Z}_{2}\right)$, where $[N \cap T]$ is the $\mathbb{Z}_{2}$-homology class represented by the $(n-3)$-dimensional submanifold $N \cap T$.

Moreover, given $r$ we can choose $\underline{c}$ such that

4. The normal geodesic tubular neighborhood of $N$ has width $>r$.

We have to define $P$. For this, let $\mathcal{B}_{3}=\mathcal{B} \backslash\left\{e_{2}\right\}$ and $\mathbf{G}_{3}=R_{k / \mathbb{Q}} \mathbf{G}_{3}^{\prime}$ where $\mathbf{G}_{3}^{\prime}$ denotes the $k$-algebraic group $S O\left(f_{3}\right)$ and $f_{3}$ is the restriction of the form $f$ to the subspace of $E$ generated by $\mathcal{B}_{3}$. It can be verified from the results of [10], Section 2, that there is an ideal $\underline{a}_{1}$ of $\mathbb{Z}$ such that $P=X_{3} / \Phi_{3}$ is a connected closed orientable totally geodesic $(n-1)$-dimensional submanifold of $X / \Phi$, where $\Phi$ is any subgroup of finite index of $\Gamma\left(\underline{a}_{1}\right)$. Here, $\Phi_{3}=\Phi \cap \mathbf{G}_{3}(\mathbb{Q})$ and $X_{3}=\left(K \cap G_{3}\right) / G_{3}$. Note that, since $\mathcal{B}_{2} \subset \mathcal{B}_{3}$, we have that $T \subset P$.

Now take an ideal $\underline{a}^{\prime} \subset \underline{a}_{0} \cap \underline{a}_{1}$. Define $\Phi^{\prime}=\Phi\left(\underline{a}^{\prime}, \underline{b}^{\prime}, \underline{b}^{\prime}\right)$ where $\underline{b}^{\prime}=\underline{b}\left(\underline{a}^{\prime}\right)$. Define $\Phi_{1}^{\prime}, \Phi_{2}^{\prime}, \Phi_{3}^{\prime}, M^{\prime}, N^{\prime}, T^{\prime}, P^{\prime}$ accordingly. Then $M^{\prime}, N^{\prime}, T^{\prime}, P^{\prime}$ satisfy $1,2,3$ of the statement of the Proposition. That is, they satisfy all required properties, except the ones about the widths of the normal geodesic tubular neighborhoods. (Note that $N^{\prime}$ and $P^{\prime}$ intersect transversally because both are different connected totally geodesic hypersurfaces of $M^{\prime}$.)

By using an argument similar to the one in pp. 235-236 of [10], we can find an ideal $\underline{a}^{\prime \prime} \subset \underline{b}^{\prime}$ such that the following holds. Define $\Phi^{\prime \prime}=\Phi\left(\underline{a}^{\prime \prime}, \underline{b}^{\prime \prime}, \underline{b}^{\prime \prime}\right)$ where $\underline{b}^{\prime \prime}=\underline{b}\left(\underline{a}^{\prime \prime}\right)$. Define $\Phi_{1}^{\prime \prime}, \Phi_{2}^{\prime \prime}, \Phi_{3}^{\prime \prime}, M^{\prime \prime}, N^{\prime \prime}, T^{\prime \prime}, P^{\prime \prime}$ accordingly. Then we can choose $\underline{a}^{\prime \prime}$ such that the normal geodesic tubular neighborhood of $P^{\prime \prime} \cap N^{\prime \prime}$ is larger that $s$. Note that $M^{\prime \prime}, N^{\prime \prime}, T^{\prime \prime}, P^{\prime \prime}$ also satisfy $1,2,3$ of the statement of the Proposition. (Here we required $\underline{a}^{\prime \prime} \subset \underline{b}^{\prime}$ so that $\Phi^{\prime \prime}$ is a subgroup of $\Phi^{\prime}$; it follows that $M^{\prime \prime} \rightarrow M^{\prime}$ is a finite cover.)

Finally, choose $\underline{c} \subset \underline{b}^{\prime \prime}$ as in [10], pp. 235-236, such that the following holds. Define $\Phi=\Phi\left(\underline{a}^{\prime \prime}, \underline{b}^{\prime \prime}, \underline{c}\right)$ and define $\Phi_{1}, \Phi_{2}, \Phi_{3}, M, N, T, P$ accordingly. Then, we can choose $\underline{c}$ such that the normal geodesic tubular neighborhood of $N$ is larger than $r$. Note that we also have $M, N, T, P$ satisfy $1,2,3$ of the statement of the Proposition and that the normal geodesic tubular neighborhood of $P \cap N$ is larger than $s$. (Note also that $N=N^{\prime \prime}$.) Then $M, N, T, P$ satisfy $1,2,3,4$ of the statement of the Proposition. This completes the proof of the Proposition.

Remark 2.1. To prove the assertion in the Remark 1.9, just replace the roles of $N$ and $T$ in the proof above. 
We now recall the construction of branched covers and introduce some notation (for more details, see [22]). Let $M$ be a hyperbolic manifold of dimension $n$ and let $R$ and $Q$ be compact totally geodesic submanifolds of $M$ of dimensions $n-1$ and $n-2$ with $\partial Q=R$. Assume that the normal bundle of $Q$ is trivial. The $i$-branched cover $M(i)$ of $M$ with respect to $(Q, R)$ is obtained in the following way. Cut $M$ along $Q$ to produce a manifold $M^{\prime}$. Since the normal bundle of $Q$ is trivial, $M^{\prime}$ contains two copies of $Q$ that intersect in $R$. Label these copies $Q_{0}$ and $Q_{1}$. Take now $i$ copies of $M^{\prime}$ and identify $Q_{1}$ of the first copy of $M^{\prime}$ with $Q_{0}$ of the second copy of $M^{\prime}$, and so on. At the end identify $Q_{1}$ of the last copy of $M^{\prime}$ with $Q_{0}$ of the first copy of $M^{\prime}$. The resulting manifold is the $i$-branched cover of $M$ with respect to $(Q, R)$. The branched cover $M(i)$ contains $i$ copies of $Q$. The union of these copies is called the $i$-book of $M(i)$. These copies intersect in a (unique) copy of $R$. Hence we consider $R$ as a submanifold of $M(i)$. There is a projection map $\pi: M(i) \rightarrow M$, which restricted to each copy of $M^{\prime}$ in $M(i)$ is just the identification map (identify back the two copies of $Q$ ). Note that if $R=\emptyset$, then $\pi: M(i) \rightarrow M$ is an $i$-sheeted (ordinary) covering space. In general, $\pi: M(i) \backslash R \rightarrow M \backslash R$ is also an $i$-sheeted covering space. Finally, note that there is an action of $\mathbb{Z}_{i}$, the cyclic group of order $i$, on $M(i)$ that "rotates" the copies of $M^{\prime}$ around $R$

Remark 2.2. We present an alternative construction of the $i$-sheeted cover of $M$ with respect to $(Q, R)$ that has an action of the dihedral group $D_{i}$ of order $2 i$. We use the same notation as above. Now take $2 i$ copies of $M^{\prime}$. Identify $Q_{1}$ of the first copy with $Q_{1}$ of the second copy, then identify $Q_{0}$ of the second copy with $Q_{0}$ of the second copy of $M^{\prime}$, and so on. We denote this resulting manifold by $M[i]$. Then $D_{i}$ acts on $M[i]$ in the obvious way. Note that we can also consider $R$ contained in $M[i]$ as before.

Gromov and Thurston proved that $M(i)$ admits a Riemannian metric with sectional curvatures equal to -1 outside a normal geodesic tubular neighborhood of $R$ of width $s$, and sectional curvatures in the interval $[-1-\epsilon,-1]$, where $0<\epsilon<\frac{(10 \log 2 i)^{2}}{s^{2}}$, inside a normal geodesic tubular neighborhood of $R$. The $\mathbb{Z}_{i}$-action on $M(i)$ (with this Riemannian metric) is by isometries.

Remark 2.3. It is a simple matter to verify that $M[i]$ (see remark 2.2) also admits a Riemannian metric with sectional curvatures equal to -1 outside a normal geodesic tubular neighborhood of $R$ of width $s$, and sectional curvatures in the interval $[-1-\epsilon,-1]$, where $0<\epsilon<\frac{(10 \log 4 i)^{2}}{s^{2}}$, inside a 
normal geodesic tubular neighborhood of $R$. Also the $D_{i}$-action on $M[i]$ is by isometries.

Gromov and Thurston also proved that, for large $i$ (how large depending on $M$ and $R$ ), $M(i)$ does not have the homotopy type of a closed locally symmetric space (they mention that probably all $M(i), i>1$, do not have the homotopy type of a closed locally symmetric space). For the proofs of the Theorems we will need the following stronger (and more technical) result, proved also by Gromov and Thurston in Section 3.5 of [14] (p. 6):

2.1. Let $M_{s}, s=1,2, \ldots$ be a sequence of closed $n$-dimensional hyperbolic manifolds, $n \geq 4$, and $R_{s}$ a closed codimension 2 totally geodesic submanifold of $M_{s}$ with trivial normal bundle that bounds a compact codimension 1 totally geodesic submanifold $Q_{s}$ of $M_{s}$. Assume that the width of the normal geodesic tubular neighborhood of $R_{s}$ is larger than $s$.

Then, there is a sequence of branched covers $M_{s}\left(i_{s}\right)$ of $M_{s}$, with respect to $\left(Q_{s}, R_{s}\right)$, satisfying the following property:

Given $\epsilon>0$ there is a $s_{0}$, such that for all $s \geq s_{0}$ we have:

1. $M_{s}\left(i_{s}\right)$ does not have the homotopy type of a closed locally symmetric space.

2. $M_{s}\left(i_{s}\right)$ admits a metric with sectional curvatures in $[-1-\epsilon,-1]$.

Remark 2.4. 1. Let $M$ be a closed negatively curved manifold of dimension $\neq 3,4$. Farrell-Jones Rigidity Theorem [7] implies that $M$ has the homotopy type of a closed locally symmetric space if and only if $M$ admits a negatively curved locally symmetric differentiable structure, i.e. a differentiable structure that supports a negatively curved locally symmetric Riemannian metric.

2. If a closed strictly $1 / 4$-pinched negatively curved Riemannian manifold is homotopically equivalent to a closed locally symmetric space $N$, then $N$ is a (real) hyerbolic manifold. This is a consequence of [16], [17], [20] and [29], and can also be seen by comparing Pontryagin classes and using a known case of Novikov's Conjecture due to Mishchenko [19].

3. We can give an altenative construction of pinched negatively curved closed manifolds that are not homotopy equivalent to a closed locally symmetric space, that do not use the results of 3.5 of [14]. Consider $M_{s}, Q_{s}$ and $R_{s}$ as in 2.1 above. To simplify our notation, we drop the subindex " $s$ ". We show that $M[2]$ or $M[2](2)$ satisfy 1 and 2 of 2.1. 
In fact (see remark 2.2), both $M[2]$ and $M[2](2)$ satisfy 2, for $s$ large enough. If $M[2]$ is not homotopically equivalent to a closed hyperbolic manifold, we are done. Suppose that $M[2]$ is homotopically equivalent to a closed hyperbolic manifold. Then there is a homotopy equivalence $f: \mathrm{M}[2] \rightarrow N$, where $N$ is a closed hyperbolic manifold. We show then that $M[2](2)$ is not homotopy equivalent to a closed locally symmetric space. Note that $D_{2}=\mathbb{Z}_{2} \oplus \mathbb{Z}_{2}$ acts on $M[2]$ and the fixed point sets of the three non-zero involutions are codimension 1 submanifolds $H_{1}$, $H_{2}$ and the codimension 2 submanifold $R=H_{1} \cap H_{2}$. Note that $H_{i}$ is homeomorphic to the double of $Q$ along its boundary $R$. Identify $Q$ with one of the two copies (of $Q$ ) that form, say, $H_{1}$. Note also that $H_{1}$ and $H_{2}$ split $M[2]$ in four pieces, each homeomorphic to $M^{\prime}$, where $M^{\prime}$ is obtained from $M$ by cutting along $Q$. Now, by Theorem 11 of [26], $D_{2}$ acts by isometries on $N$ and $f$ is homotopic to a $D_{2}$ equivariant map $g$. Pansu in [22] shows that $N$ also splits in four homeomorphic pieces and $g$ induces an isomorphism of fundamental groups from each copy of $M^{\prime}$ in $M[2]$ to each of the four pieces of $N$ (and these isomorphisms are also isomorphisms when restricted to the boundaries). Using this, it is not difficult to construct a $D_{2}$-equivariant map $h$ homotopic to $f$ such that $h$ maps each copy of $M^{\prime}$ to each of the four pieces of $N$. In particular, $Q^{\prime}=h(Q), R^{\prime}=h(R)$ are totally geodesic submanifolds of $N$ and $\partial Q^{\prime}=R^{\prime}$. This $h$ can be lifted to give a homotopy equivalence between $M[2](2)$ and $N(2)$, where the 2 -branched covers are taken with respect to $(Q, R)$ and $\left(Q^{\prime}, R^{\prime}\right)$, respectively. But 3.6 of [14] implies that $N(2)$ (and consequently, also $M[2](2))$ is not homotopy equivalent to a closed locally symmetric space, that is, $M[2](2)$ satisfies 1 and 2 of 2.1 .

Throughout the proofs of all Theorems in this paper, we can replace the use of $M(i)$ (in 2.1 above) by $M[2]$ or $M[2](2)$ (whichever satisfies 1 of 2.1.), and the proofs remain valid.

Proof. of Theorem 1.1 Fix $\epsilon>0$. For each $s=1,2,3, \ldots$ let $M_{s}^{\prime}, N_{s}^{\prime}, P_{s}^{\prime}$ and $T_{s}^{\prime}$ be as in Proposition 1.8, with the width of the normal geodesic tubular neighborhood of $N_{s}^{\prime} \cap P_{s}^{\prime}$ larger than $s$ and with large $r$ (how large will be determined in a moment; note that, by item 2 of Proposition 1.8, $N_{s}^{\prime}$ does not depend on $r$ ). We assume also $r>2 s$. To alleviate the notation we will drop the subindex " $s$ " whenever this causes no confusion. Write $U^{\prime}=N^{\prime} \cap T^{\prime}$. By item 3 of Proposition $1.8 c^{\prime}=\operatorname{Dual}\left(U^{\prime}\right) \neq 0 \in H^{3}\left(M^{\prime}, \mathbb{Z}_{2}\right)$. Then we have a smooth structure $\Sigma^{\prime}$ on $M^{\prime}$ such that its corresponding $P L$ structure 
corresponds to $c^{\prime}$. (We choose this correspondence to assign the hyperbolic differentiable structure to $0 \in H^{3}\left(M^{\prime}, \mathbb{Z}_{2}\right)$, see [21]. It follows that $\Sigma^{\prime}$ is not $P L$-concordant to the hyperbolic differentiable structure, see [18].) Choose $r$ large enough so that $\left(M^{\prime}, \Sigma^{\prime}\right)$ admits a Riemannian metric with sectional curvatures $\epsilon$-close to -1 , see $[21]$ ( $r$ depends on $s$ and $\epsilon$ ). The important point here is the following. Identify (a piece of) the tubular neighborhood of $N^{\prime}$ with $N^{\prime} \times[-r, r]$. Then outside $V^{\prime}=N^{\prime} \times\left[-r,-\frac{r}{2}\right]$ we have that $\Sigma^{\prime}$ coincides with the hyperbolic differentiable structure and the $\epsilon$-pinched metric, mentioned above, is hyperbolic, see [21]. That is, the change of the differentiable structure and the change of the metric only happen inside $V^{\prime}$. Note that the distance between $V^{\prime}$ and $N^{\prime}$ is $\frac{r}{2}>s$.

Remark 2.5. Since the differentiable structure $\Sigma^{\prime}$ is not $P L$-concordant to the hyperbolic differentiable structure, we have that the identity map $M^{\prime} \rightarrow\left(M^{\prime}, \Sigma^{\prime}\right)$ is not homotopic to a $P L$ homeomorphism. More generally, if $M$ is any closed non positively curved manifold of dimension $\neq 3,4$, and $\Sigma$ is a differentiable structure not $P L$-concordant to the given non positively curved differentiable structure then the identity map $M \rightarrow(M, \Sigma)$ is not homotopic to a $P L$ homeomorphism. To see this, suppose that $h: M \times[0,1] \rightarrow M$ is a homotopy from $i d_{M}$ to a $P L$ homeomorphism. Then, by Farrell-Jones Rigidity Theorem [7], the map $H^{\prime}(x, t)=(h(x, t), t)$ is homotopic to a homeomorphism $H: M \times[0,1] \rightarrow M \times[0,1]$ with $H_{0}=i d_{M}$ and $H_{1}$ equal to the $P L$ homeomorphism above. It follows that $\Sigma$ is $P L$ concordant to the given non positively curved differentiable structure.

From item 3 of Proposition 1.8 , it follows that $0 \neq\left[N^{\prime}\right] \in H_{n-1}\left(M^{\prime}, \mathbb{Z}_{2}\right)$. Let $M$ be the double cover of $M^{\prime}$ with respect to $N^{\prime}$, that is, with respect to the kernel of the map $\pi_{1} M^{\prime} \rightarrow \mathbb{Z}_{2}$ given by $\alpha \mapsto \operatorname{Dual}\left(N^{\prime}\right)[\alpha]$. Let $p: M \rightarrow$ $M^{\prime}$ be the covering projection. Note that $M$ consists of two copies $A, B$ of the manifold obtained from $M^{\prime}$ by cutting along $N^{\prime}$. $A$ and $B$ intersect in two copies of $N^{\prime}$. Denote one of these copies by $N$. Let $T=p^{-1}\left(T^{\prime}\right)$. Write $U=N \cap T$. Then $\left.p\right|_{U}: U \rightarrow U^{\prime}$ is a homeomorphism (in fact an isometry). Hence $0 \neq[U] \in H_{n-3}(M)$. Hence, as before, $c=\operatorname{Dual}(U) \neq$ $0 \in H^{3}\left(M, \mathbb{Z}_{2}\right)$. Also, the normal geodesic tubular neighborhood of $N$ is as large as the normal geodesic tubular neighborhood of $N^{\prime}$ and let $V$ be the piece of $p^{-1}\left(V^{\prime}\right)$ that lies in the $r$ normal geodesic tubular neighborhood of $N$. Also we assume that, say, $V \subset A$. Note that $V$ is a piece, of width $\frac{r}{2}$, of the normal geodesic tubular neighborhood of $N$ which is at a distance $\frac{r}{2}>s$ from $B$. 
Let $P=p^{-1}\left(P^{\prime}\right), Q=P \cap B$ and $R=\partial Q$. Note that $R$ consists of two copies of $N^{\prime} \cap P^{\prime}$ and that the width of the normal geodesic tubular neighborhood of $R$ is larger than $s$. Note also that the distance between $V$ and $Q$ is $>s$.

So far, we have obtained (we write back the subindex " $s$ ") sequences $M_{s}$, $N_{s}, T_{s}, R_{s}, Q_{s}, V_{s}, U_{s}=N_{s} \cap T_{s}, c_{s}$ satisfying:

a. $M_{s}$ is a closed $n$-dimensional hyperbolic manifold and $N_{s}$ is a closed codimension one totally geodesic submanifold of $M_{s} . U_{s}$ is a closed codimension three totally geodesic submanifold of $M_{s}$ and $U_{s} \subset N_{s}$.

b. $R_{s}$ is a closed codimension 2 totally geodesic submanifold of $M_{s}$ that bounds the compact codimension 1 totally geodesic submanifold $Q_{s}$. The width of the normal geodesic tubular neighborhood of $R_{s}$ is larger than $s$.

c. $V_{s}$ is a piece of the tubular neighborhood of $N_{s}$ and $V_{s}$ is at a distance $>s$ from $Q_{s}$.

d. $c_{s}=\operatorname{Dual}\left(U_{s}\right)$ is a non zero cohomology class. As for $M_{s}^{\prime}, M_{s}$, with the smooth structure $\Sigma_{s}$ whose corresponding $P L$ structure corresponds to $c_{s}$, admits a Riemannian metric with sectional curvatures $\epsilon$-close to -1 . Outside $V_{s}$ this $\epsilon$-pinched to -1 metric is hyperbolic.

By 2.1, there is a $s_{0}$, such that for all $s \geq s_{0}$, we have:

1. $M_{s}\left(i_{s}\right)$ does not have the homotopy type of a locally symmetric space.

2. $M_{s}\left(i_{s}\right)$ admits a metric with sectional curvatures in $[-1-\epsilon,-1]$. This metric is hyperbolic outside the normal geodesic tubular neighborhood of width $s$ of $R_{s}$.

Write $\left(M_{1}\right)_{s}=M_{s}\left(i_{s}\right)$ and we again drop the subindex " $s$ ". Let $\pi: M_{1} \rightarrow$ $M$ be the ramified projection. Choose a copy of $M \backslash(Q \backslash R)$ in $M_{1}$. Hence, we then can find $N_{1}, V_{1}, U_{1}$ contained in this copy such that:

$$
\left.\pi\right|_{N_{1}}: N_{1} \rightarrow N,\left.\pi\right|_{V_{1}}: V_{1} \rightarrow V,\left.\pi\right|_{U_{1}}: U_{1} \rightarrow U \text { are homeomorphisms. }
$$

Since $U$ does not bound, it follows that $c_{1}=\operatorname{Dual}\left(U_{1}\right) \neq 0 \in$ $H^{3}\left(M_{1}, \mathbb{Z}_{2}\right)$. Then we have a smooth structure $\Sigma_{1}$ on $M_{1}$ such that its corresponding $P L$ structure corresponds to $c_{1}$. (Again, we choose this correspondence to assign the branched differentiable structure to $0 \in H^{3}\left(M^{\prime}, \mathbb{Z}_{2}\right)$.) Hence $\Sigma_{1}$ is not $P L$-concordant to the branched differentiable structure. 
But $M_{2}=\left(M_{1}, \Sigma_{1}\right)$ admits a Riemannian metric with sectional curvatures $\epsilon$-close to -1 such that outside $V_{1}$ this metric coincides with the metric of item 2 above. It follows then that the identity map $i d: M_{1} \rightarrow M_{2}$ is a homotopy equivalence between $\epsilon$-pinched to -1 closed manifolds which is not homotopic to a $P L$ homeomorphism (see the last remark above) and $M_{1}$ and $M_{2}$ do not have the homotopy type of a locally symmetric space. This proves Theorem 1.1.

Corollary 1.3 can be directly deduced from Theorem 1.1 and the $C^{\infty}-$ Hauptvermutung of Scharlemann and Siebenmann [24]:

Proof. of Corollary 1.3 By Theorem 1.1, we have In every dimension $n \geq 6$, there is a pair of closed negatively curved $n$ dimensional manifolds $M_{1}$ and $M_{2}$ and a homeomorphism $f: M_{1} \rightarrow M_{2}$ that satisfy (2) and (3) of the statement of Corollary 1.3, and $f$ is not homotopic to a PL homeomorphism.

Let $h: M_{1} \rightarrow M_{2}$ be the (unique) harmonic map homotopic to $f$. We have to prove that $h$ is not one-to-one. But this follows by just applying the following result of M. Scharlemann and L. Siebenmann [24]

A smooth homeomorphism between closed smooth manifolds of dimension $\geq$ 6 is homotopic to a PL-homeomorphism.

(Smooth homeomorphisms are not necessarily diffeomorphisms. A simple example is given by the smooth homeomorphism $f: \mathbb{R} \rightarrow \mathbb{R}, f(x)=x^{3}$.)

Thus, the harmonic map $h$ cannot be a homeomorphism (equivalently, cannot be one-to one) because $f$ is not homotopic to a $P L$ homeomorphism. This proves Corollary 1.3.

Before we prove Theorem 1.4, we need a remark about coverings of branched covers. Let $p: M \rightarrow M^{\prime}$ be a cover, where $M$ and $M^{\prime}$ are hyperbolic manifolds. Let $R$ and $R^{\prime}$ be closed codimension two totally geodesic submanifolds of $M$ and $M^{\prime}$, respectively, that bound closed codimension one totally geodesic submanifolds $Q$ and $Q^{\prime}$ of $M$ and $M^{\prime}$, respectively. Assume $Q=p^{-1}\left(Q^{\prime}\right)$ and $R=p^{-1}\left(R^{\prime}\right)$. Then, for any $i$, we can use $p$ to construct a cover $q: M(i) \rightarrow M^{\prime}(i)$, where $M(i)$ and $M^{\prime}(i)$ are the $i$-branched covers of $M$ and $M^{\prime}$ with respect to $(Q, R)$ and $\left(Q^{\prime}, R^{\prime}\right)$ respectively. The covers $p$ and $q$ fit in the following commutative square:

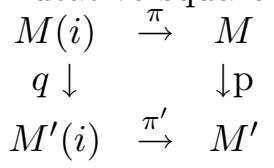

If $p$ is a $\ell$-sheeted cover, then so is $q$. If $p$ is a regular cover, so is $q$. We now prove Theorem 1.4. 
Proof. of Theorem 1.4 Let $M^{\prime}, N^{\prime}, T^{\prime}, R^{\prime}, Q^{\prime}, V^{\prime}, U^{\prime}, c^{\prime}, \Sigma^{\prime}$ be as in the proof of Theorem 1.1 (we are dropping the subindex " $s$ " and introducing a prime on each symbol). These objects satisfy properties a, b, c, d in the proof of Theorem 1.1. We assume also $T^{\prime} \subset P^{\prime}$ (see Remark 1.9). Let also $M_{1}^{\prime}, N_{1}^{\prime}, V_{1}^{\prime}, U_{1}^{\prime}, c_{1}^{\prime}, \pi^{\prime}, \Sigma_{1}^{\prime}, M_{2}^{\prime}$ be as in the proof of Theorem 1.1 (we are also dropping the subindex " $s$ " and introducing a prime on each symbol). We have that $M_{1}^{\prime}$ satisfies properties a and $\mathrm{b}$ in the proof of Theorem 1.1 (assuming $s$ large enough).

Let $p: M \rightarrow M^{\prime}$ be the double cover of $M^{\prime}$ with respect to $N^{\prime}$. Note that $M$ consists of two copies $D$, and $E$ of the manifold obtained from $M^{\prime}$ by cutting along $N^{\prime}$. D and $E$ intersect in two copies of $N^{\prime}$. Let $\Sigma=p^{*} \Sigma^{\prime}$. We know from [10] that the identity $M \rightarrow(M, \Sigma)$ is now homotopic to a diffeomorphism $f: M \rightarrow(M, \Sigma)$, but the unique harmonic map $h$ homotopic to $f$ (or to the identity) is not one-to-one. This is because the $P L$ structure corresponding to $\Sigma$ corresponds to $c=p^{*}\left(c^{\prime}\right)$ which vanishes. (The cohomology class $c$ vanishes because it is dual to $p^{-1}\left(U^{\prime}\right)$, which is the boundary of $D \cap p^{-1}\left(T^{\prime}\right)$.)

Let $M_{1}=M(i)$. Since we also have (by definition) $M_{1}^{\prime}=M^{\prime}(i)$, the commutative square given just before this proof becomes:

$$
\begin{array}{ccc}
M_{1} & \stackrel{\pi}{\rightarrow} & M \\
q \downarrow & & \downarrow p \\
M_{1}^{\prime} & \stackrel{\pi^{\prime}}{\rightarrow} & M^{\prime}
\end{array}
$$

Define $\sigma_{1}=q^{*} \Sigma_{1}^{\prime}, M_{2}=\left(M_{1}, \Sigma_{1}\right), c_{1}=q^{*} c_{1}^{\prime}$ and $U_{1}=q^{-1}\left(U_{1}^{\prime}\right)$. Note that:

(i) $q: M_{2} \rightarrow M_{2}^{\prime}$ is a smooth cover.

(ii) $M_{1}^{\prime}=M_{1} / F$, where $F=\{i d, \phi\} \cong \mathbb{Z}_{2}$ and $\phi: M_{1} \rightarrow M_{1}$ is the unique non trivial covering transformation.

(iii) The $P L$ structure corresponding to $\Sigma_{1}$ corresponds to $c_{1}$.

(iv) $c_{1}$ is dual to $U_{1}$.

Claim. $M_{1}$ is not homotopy equivalent to a locally symmetric space.

If $M_{1}$ supports a negatively curved locally symmetric differentiable structure (see item 1 of Remark 2.4) then, by Mostow's Rigidity Theorem, $\phi$ can be realized by an isometry. It follows that $M_{1}^{\prime}$ admits a negatively curved locally symmetric differentiable structure. This is a contradiction. This proves the claim. 
Now, since $p: M_{1} \rightarrow M_{1}^{\prime}$ and $q: M_{2} \rightarrow M_{2}^{\prime}$ are smooth covers, we have that both $M_{1}, M_{2}$ admit $\epsilon$-pinched to -1 Riemannian metrics.

It remains to prove that $\Sigma_{1}$ is $D I F F$-concordant to the differentiable structure of $M_{1}$. To be able to repeat the argument given in [10], pp. 230233 , we need to prove that the cohomology class $c_{1}$ vanishes. Equivalently, we need to prove that $U_{1}$ bounds. Recall that $U^{\prime}=T^{\prime} \cap N^{\prime}$. We assume that $T^{\prime} \subset P^{\prime}$ (see remark after the statement of the Proposition). Then we can also find $T_{1}^{\prime}$ in one of the copies that form $M_{1}^{\prime}$ such that $\left.\pi^{\prime}\right|_{T_{1}^{\prime}}: T_{1}^{\prime} \rightarrow T^{\prime}$ is a homeomorphism and $U_{1}^{\prime} \subset T_{1}^{\prime}$. Then $U_{1}^{\prime}=T_{1}^{\prime} \cap N_{1}^{\prime}$. Let $T_{1}=q^{-1}\left(T_{1}^{\prime}\right)$. A simple geometric argument then shows that $U_{1}=\partial\left(T_{1} \cap D_{1}\right)$, where $D_{1}$ lies in the same copy where $T_{1}$ lies and $\pi\left(D_{1}\right)=D$. This proves Theorem 1.4.

Proof. of Theorem 1.5 We use all notation from the proof of Theorem 1.4, with the following changes.

1. Now, we assume that the width of the normal geodesic tubular neighborhood of $T^{\prime}$ (not of $N^{\prime}$ ) is larger than $r$ (see remark after the statement of the Proposition). It follows that the width of the normal geodesic tubular neighborhood of $T$ is also larger than $r$.

2. The changes of structure and metric happen now in a piece of the normal geodesic tubular neighborhood of $T^{\prime}$ and $T$ : since the normal bundle of $T^{\prime}$ is trivial, we have $\mathcal{N}_{r}\left(T^{\prime}\right) \backslash T^{\prime}$ can be identified with $T^{\prime} \times$ $\mathbb{S}^{1} \times(0, r)$, where $\mathcal{N}_{r}\left(T^{\prime}\right)$ is the normal geodesic tubular neighborhood of $T^{\prime}$ of width $r$. In [11] it is shown that we can take now $V^{\prime}=$ $T^{\prime} \times I \times\left(0, \frac{r}{2}\right)$, where $I \subset \mathbb{S}^{1}$ is any non-trivial interval. That is, outside $V^{\prime}$, the differentiable structure $\Sigma^{\prime}$ coincides with the hyperbolic differentiable structure, and the metric is hyperbolic. Note that, by choosing $I$ properly, we have that $V^{\prime}$ does not intersect $P^{\prime}$.

3. Now $p: M \rightarrow M^{\prime}$ denotes the finite sheeted cover given in [11]. Again, we have that $\Sigma$ is now DIFF-concordant to the hyperbolic differentiable structure. The new feature now is that the metric pulled back from $\left(M^{\prime}, \Sigma^{\prime}\right)$ can be deformed to the hyperbolic one and all this deformation happens inside $V$.

Now, since $V$ does not intersect $P$, we can also deform the metric of $M_{1}$ to the metric pulled back from $M_{2}$, and all this happens inside $V_{1}$. This is the main ingredient needed for the proof. The rest follows exactly as in [11]. This completes the proof of Theorem 1.5. 
Proof. of Theorem 1.7. The proof follows the ideas of the proofs of Theorem and Corollary 1.3 in [12]. We use all objects in the proof of Theorem 1.5 above, but change the notation a bit to match the notation in [12]. From the proof above we have a homotopy commutative diagram:

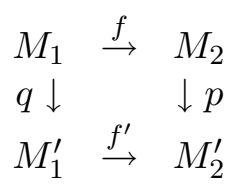

where $f$ is a diffeomorphism and $f^{\prime}$ is not homotopic to a $P L$ homeomorphism. Recall that $f^{\prime}$ is the identity and $f$ is homotopic to the identity (the underlying topological manifolds of $M_{2}^{\prime}=\left(M_{1}^{\prime}, \Sigma^{\prime}\right)$ and $M_{2}=\left(M_{1}, \Sigma\right)$ are $M_{1}^{\prime}$ and $M_{1}$ respectively). We now change the notation to match the one in [12]. First identify $M_{1}$ with $M_{2}$ via $f$ and write $N$. Then write $M_{0}$ for $M_{1}^{\prime}$, $M_{1}$ for $M_{2}^{\prime}, f$ for $f^{\prime}, p_{0}$ for $q$ and $p_{1}$ for $p f$. The diagram above becomes:

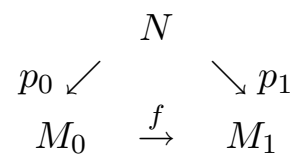

As in [12], we have then that $M_{0}, M_{1}, N$, are smooth closed manifolds of dimension $n>10$, that admit Riemannian metrics $g_{0}, g_{1}$ on $M_{0}$ and $M_{1}$, respectively, and smooth regular finite covers $p_{0}: N \rightarrow M_{0}, p_{1}: N \rightarrow M_{1}$ such that:

(1) The map $f$ is not homotopic to a $P L$-homeomorphism.

(2) and $g_{0}$ and $g_{1}$ have sectional curvatures in $[-1-\epsilon,-1+\epsilon]$.

(3) There is a $C^{\infty}$ family of $C^{\infty}$ Riemannian metrics $h_{s}$ on $N, 0 \leq s \leq 1$, with $h_{0}=p_{0}^{*} g_{0}$, and $h_{1}=p_{1}^{*} g_{1}$, such that every $h_{s}$ has sectional curvatures in $[-1-\epsilon,-1+\epsilon]$.

Let $G_{i} \subset \operatorname{Diff}(N)$, be (finite) subgroups of the group $\operatorname{Diff}(N)$, of all self-diffeomorphisms of $N$, such that $N / G_{i}=M_{i}, i=0,1$. It was shown in [10], [11], that $G_{0}$ and $G_{1}$ are conjugate in $T o p N$, via a homeomorphism homotopic to $i d_{N}$; hence $\gamma G_{0}=\gamma G_{1}$, where $\gamma: \operatorname{Diff}(N) \rightarrow \operatorname{Out}\left(\pi_{1} N\right)$ is the map described in the proof of Corollary 1.3 in [12]. Note that $G_{i} \subset$ $\operatorname{Iso}\left(N, h_{i}\right)$, where $\operatorname{Iso}\left(N, h_{i}\right) \subset \operatorname{Diff}(N)$ is the subgroup consisting of all isometries of the negatively curved manifold $\left(N, h_{i}\right)$.

If the Ricci flow does not converge smoothly to a negatively curved metric for some $h_{s}$, we are done. So, let us assume that the Ricci flow converges smoothly to a negatively curved metric for all $h_{s}$. We will show a contradiction. Write $h_{s, t}$, for the Ricci flow starting at 
$h_{s, 0}=h_{s}, 0 \leq t<\infty$, converging to the negatively curved Einstein metric $j_{s}$. Using the same argument as the one given in the proof of Theorem of [12], we get that all $j_{s}$ are equal (modulo diffeomorphism and rescaling). Moreover, there is a diffeomorphism $\phi: N \rightarrow N$ homotopic to the identity such that $j_{0}$ is equal to $\phi^{*} j_{1}$ up to scaling (see also the proof of Corollary 1.3 in [12]). It follows that $G_{1}$ is conjugate in $\operatorname{Diff}(N)$ to a subgroup of $\operatorname{Iso}\left(N, j_{0}\right)$ via a diffeomorphism $\phi$ homotopic to $i d_{N}$; i.e. $\phi^{-1} G_{1} \phi \subset I s o\left(N, j_{0}\right)$. Note that $\gamma\left(\phi^{-1} G_{1} \phi\right)=\gamma\left(G_{1}\right)$ since $\phi \sim i d_{N}$; hence $\gamma\left(\phi^{-1} G_{1} \phi\right)=\gamma\left(G_{0}\right)$. This implies that $\phi^{-1} G_{1} \phi=G_{0}$ since both $\phi^{-1} G_{1} \phi$ and $G_{0}$ are subgroups of $I s o\left(N, j_{0}\right)$ and Borel-Conner-Raymond showed (see [4], p. 43) that $\gamma$ restricted to compact subgroups of $\operatorname{Diff}(N)$ is monic. (Recall that $N$ is aspherical and the center of $\pi_{1}(N)$ is trivial.) It follows that $\phi$ induces a diffeomorphism $\varphi$ between $M_{0}=N / G_{0}$ and $M_{1}=N / G_{1}$. To find a contradiction, we have to prove that $\varphi$ is homotopic to the identity. Since all manifolds here are aspherical, it is enough to prove that the induced map $\varphi_{*}$ at the fundamental group level is the identity. Note that, since $\phi_{*}$ is the identity, we have that $\left.\varphi_{*}\right|_{\pi_{1} N}$ is the identity. Then, the fact that $\varphi_{*}$ is also the identity follows from the next Lemma:

Lemma 2.6. Let $\Gamma$ be the fundamental group of a closed negatively curved manifold. Let $H$ be a subgroup of finite index of $\Gamma$ and let $\alpha: \Gamma \rightarrow \Gamma$ be an isomorphism whose restriction to $H$ is the identity. Then $\alpha$ is also the identity.

Proof. Let $x \in \Gamma$. Since $H$ has finite index in $\Gamma$ we have that there is a positive integer $n$ such that $x^{n} \in H$. Hence $\alpha\left(x^{n}\right)=x^{n}$. Therefore, $(\alpha(x))^{n}=x^{n}$. But $n$-roots are unique in $\Gamma$, thus $\alpha(x)=x$. This proves the Lemma.

\section{Acknowledgement.}

We wish to thank J-F. Lafont for the helpful discussions we had with him while writing this paper. 


\section{References.}

[1] S. I. Al'ber, Spaces of mappings into manifold of negative curvature, Dokl. Akad. Nauk USSR 168 (1968), 13-16.

[2] S. Ardanza, PhD Thesis, Binghamton University (2000)

[3] G. Besson, G. Courtois and S. Gallot, Minimal entropy and Mostow's rigidity Theorems, Ergodic Theory \& Dynam. Sys. 16 (1996), 623-649.

[4] P. E. Conner and F. Raymond, Deforming homotopy equivalences to homeomorphisms in aspherical manifolds, Bull. AMS, 83 (1977), 3685.

[5] J. Eells and J. H. Sampson, Harmonic mappings of Riemannian manifolds, Amer. J. Math. 86 (1964), 109-160.

[6] F. T. Farrell and L. E. Jones, Negatively curved manifolds with exotic smooth structures, J. Amer. Math. Soc. 2 (1989), 899-908.

[7] F. T. Farrell and L. E. Jones, Rigidity in geometry and topology, Proc. of the International Congress of Mathematicians, Vol. I, II (Kyoto, 1990), Math. Soc. Japan, Tokyo (1991), 653-663.

[8] F. T. Farrell, L. E. Jones and P. Ontaneda, Hyperbolic manifolds with negatively curved exotic triangulations in dimension larger than five. Jour. Diff. Geom. 48 (1998), 319-322.

[9] F. T. Farrell, L. E. Jones and P. Ontaneda, Examples of nonhomeomorphic harmonic maps between negatively curved manifolds, Bull. London Math. Soc. 30 (1998), 295-296.

[10] F. T. Farrell, P. Ontaneda and M. S. Raghunathan, Non-univalent harmonic maps homotopic to diffeomorphisms, Jour. Diff. Geom. 54 (2000), 227-253.

[11] F. T. Farrell and P. Ontaneda, Cellular harmonic maps which are not diffeomorphisms, Invent. Math. 158 (2004), 497-513.

[12] F. T. Farrell and P. Ontaneda, A caveat on the convergence of the Ricci flow for negatively curved manifolds, Asian J. of Math. 9(2005), 401406. 
[13] F. T. Farrell and P. Ontaneda, Exotic structures and the limitations of certain analytic methods in geometry, Asian J. Math. 8, n. 4 (2004), 639-652.

[14] M. Gromov and W. Thurston, Pinching constants for hyperbolic manifolds, Invent. Math. 89 (1987), 1-12.

[15] P. Hartman, On homotopic harmonic maps, Canad. J. Math. 19 (1967), 673-687.

[16] L. Hernández, Kähler manifolds and 1/4-pinching, Duke Math. J. 62 (1991), 601-611.

[17] J. Jost and S.-T. Yau, Harmonic maps and superrigidity, Proc. Sympos. Pure Math. 54 (1), Amer. Math. Soc., Providence, R.I. (1993), 245-280.

[18] R. C. Kirby and L. C. Siebenmann, Foundational Essays on Topological Manifolds, Smoothings, and Triangulations, Annals of Math. Studies no.88, Princeton University Press, Princeton (1977).

[19] A.S.Mishchenko, Infinite dimensional representations of discrete groups and higher signatures, Izv. Akad. Nauk SSSR Ser. Mat. 38 (1974), 81106.

[20] N. Mok, Y.-T. Siu and S.-K. Yeung, Geometric superrigidity, Invent. Math. 113 (1993), 57-83.

[21] P. Ontaneda, Hyperbolic manifolds with negatively curved exotic triangulations in dimension six, J. Diff. Geom. 40 (1994), 7-22.

[22] Pansu, Pincement des variétés à courbure négative, Séminaire de théorie spectrale el géométrie Chambéry-Grenoble (1985-1986), 101-113.

[23] J. Sampson, Some properties and applications of harmonic mappings, Ann. Scient. Ec. Norm. Sup. 11 (1978), 211-228.

[24] M. Scharlemann and L. Siebenmann, The Hauptvermutung for smooth singular homeomorphisms, in Manifolds Tokyo 1973, Akio Hattori ed. Univ. of Tokyo Press, 85-91.

[25] R. Schoen and S.-T. Yau, On univalent harmonic maps between surfaces, Inv. Math. 44 (1978), 265-278.

[26] R. Schoen and S.-T. Yau, Compact group actions and the topology of manifolds with non-positive curvature, Topology 18 (1979), 361-380. 
[27] C. W. Stark, Surgery theory and infinite fundamental groups, Ann. of Math. Studies 145, Vol.1, 239-252.

[28] S.-T. Yau, Seminar on differential geometry, Ann.of Math. Stud., 102, Princeton Univ. Press, Princeton, NJ, 1982.

[29] S.-T. Yau and F. Zheng, Negatively 1/4-pinched Riemannian metric on a compact Kähler manifold, Invent. Math. 103 (1991), 527-535.

F. T. Farrell

SUNY, Binghamton, N.Y., 13902, U.S.A.

P. Ontaneda

SUNY, Binghamton, N.Y., 13902, U.S.A. 